\title{
Víctor L. Urquidi: insatisfacción ilustrada de un polémico economista mexicano
}

\begin{abstract}
“... si la sociedad no
convierte este destino en otro mejor, las libertades y los derechos del individuo que, en el mundo en su conjunto, hasta ahora ha disfrutado sólo una minoría, pasarán a ser un recuerdo lejano. El porvenir está férreamente unido a la Economía."
\end{abstract}

Víctor L. Urquidi, Discurso de ingreso a El Colegio Nacional, octubre de

1960.

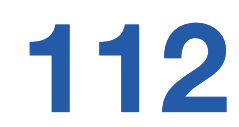

\section{Eduardo Vega López}

Profesor titular "A" de tiempo completo y director de la Facultad de Economía de la UNAM «eduardov@economia.unam.mx»

\section{Introducción} la vida pública nacional, a la influencia de sus diagnósticos económicos y propuestas de política, a escalas nacional, latinoamericana e internacional, y a su honestidad y generosidad intelectuales; Víctor L. Urquidi sigue vivo entre nosotros, al tiempo que su obra continuará animando valiosas discusiones entre economistas, sociólogos, historiadores, demógrafos y otros profesionales.

La publicación de las Obras Escogidas de Víctor L. Urquidi, por El Colegio de México a partir de 2007 , no sólo hace posible recapitular la vida y la herencia reflexiva, abarcante, polémica de este esencial economista mexicano, sino que mediante la lectura de diversos escritos allí ordenados temáticamente en sus cinco volúmenes, es posible poner en contexto histórico e institucional importantes situaciones y debates que perviven en la actualidad económica, social y ambiental del país, así como en varias de las dimensiones de la realidad internacional que continúan preocupándonos.

Como funcionario del gobierno mexicano, funcionario internacional, académico y editor de El Trimestre Económico y de diversos libros publicados por el Fondo de Cultura Económica, siempre impulsó la investigación documentada, la discusión informada y la toma de decisiones acerca de la importancia crucial de la industrialización, la modernización económica, el fortalecimiento fiscal del Estado, la administración adecuada de los desequilibrios de la balanza de pagos, la deliberada orientación del ahorro hacia la inversión pública para el desarrollo, así como acerca de la imperiosa urgencia por modificar las inercias del mercado y de las políticas que, según sus diagnósticos, lejos de erradicar la concentración excesiva de la propiedad y del ingreso nacional, la arraigaban estructural y dinámicamente. 
Detrás de su personalidad adusta y, en no pocas ocasiones, irascible, se encontraba su verdadera vocación de servicio público y solidaridad social. Guiado por su insatisfacción ilustrada con la realidad social nacional, con la política económica y demográfica vigentes, con la irresponsabilidad privada y la desatención pública relacionadas con los impactos ambientales del crecimiento económico en el país, fue un incansable constructor de instituciones, precursor de importantes debates nacionales, promotor de programas académicos sobresalientes y formador exigente de economistas.

\section{Hondura y vigencia de sus contribuciones}

En 1941, recién egresado de la London School of Economics and Political Science, a sus 22 años de edad, se integró al Departamento de Estudios

Económicos del Banco de México y, a sus 25, fue el secretario técnico de la delegación mexicana que participó en la Conferencia Monetaria y Financiera de las Naciones Unidas, realizada en Bretton Woods, New Hampshire, en julio de 1944. En ese carácter y con esas funciones, el entonces muy joven economista Víctor Luis Urquidi Bingham, redactó la propuesta de enmienda mexicana al texto que daba origen formal al Banco Internacional de Reconstrucción y Fomento (BIRF, hoy Banco Mundial). Esa exitosa enmienda, notable pieza de la diplomacia económica mexicana de entonces, argüía acerca de la necesidad y la conveniencia de que además de preocuparse por la reconstrucción económica europea y asiática, durante la ya próxima segunda posguerra, también se comprometiera el naciente BIRF con el desarrollo económico. Por la trascendencia de tal enmienda y con el ánimo de documentar, con un botón de muestra, la influencia internacional de Urquidi, al final de este breve artículo se ofrece, como anexo, la versión completa y original de la misma.

Al revisar con cuidado las actas y documentos oficiales de la referida conferencia de Bretton Woods, ${ }^{1}$ puede acreditarse que la propuesta redactada y leída por Víctor L. Urquidi el 12 de julio de 1944, en sesión formal del pleno de la Comisión II presidida por John Maynard Keynes, hacía referencia explícita a la imperiosa necesidad y conveniencia del desarrollo económico:

... we believe that the agreement we are to reach here is to be embodied in a permanent, and not in a provisional, international instrument. Therefore, it seems to us inappropriate that the document should not contain an equal emphasis on the two great purposes of the Bank, namely, to facilitate reconstruction and development. In the very short run, perhaps reconstruction will be more urgent for the world as a whole, but in the long run, Mr. Chairman - before we are all too dead, if I may say so- development must prevail if we are to sustain and increase real income everywhere. Without denying the initial importance of reconstruction, we ask you not to relegate or postpone development.

1 Proceedings and Documents of United Nations Monetary and Financial Conference, Bretton Woods, New Hampshire, july 1-22, 1944,vol. II, pp. 1175-1177, Document 306. También disponible al teclear en cualquier buscador de internet la siguiente dirección: https://fraser.stlouisfed.org/docs/publications/books/1948_state_bwood_v2.pdf. 
Después de un tenso pero por fortuna breve silencio, según el propio Urquidi refiere en un interesante artículo al respecto, ${ }^{2}$ el presidente de esa Comisión II, John Maynard Keynes, dijo:

With regard to the amendment submitted by Mexico, I should think it can be made shorter and can be adopted... (y propuso que la redacción definitiva del acuerdo correspondiente quedara como sigue)... The resources and the facilities of the Bank shall be used exclusively for the benefit of members with equitable consideration to projects for development and projects for reconstruction alike.

Con esta enmienda mexicana aprobada en las resoluciones definitivas de Bretton Woods, así como con las demás intervenciones de la delegación mexicana registradas en esa trascendente conferencia internacional, podría decirse que emergían formalmente algunas de las preocupaciones fundamentales que convergerían, poco más tarde, en lo que se conocería como inversión para el desarrollo, pieza clave del estructuralismo económico latinoamericano, lo cual, se plasmaría también en la institucionalidad de las Naciones Unidas, a partir de diciembre de 1948, con la creación de la Comisión Económica para América Latina y el Caribe (CEPAL). Tomando como referencia a Joseph Hodara, la juventud de Víctor L. Urquidi fue la única razón que impidió la posibilidad real de haber sido designado para encabezar la entonces naciente CEPAL. ${ }^{3}$

Cuatro años después de la fundación de la CEPAL y hasta 1960, Víctor L. Urquidi dirigió la sede subregional en México de dicha institución, siendo una de sus principales contribuciones el haber diseñado, activamente promovido y puesto en vigor, el Tratado Multilateral de Libre Comercio e Integración Centroamericana. Este Tratado, firmado en Tegucigalpa, Honduras, en junio de 1958, fue el antecedente fundamental para la creación formal del Mercado Común Centroamericano, en 1960.

De manera simultánea y debido a su considerable capacidad de trabajo, de 1943 a 1948, al lado de Daniel Cosío Villegas, Eduardo Villaseñor y Emigdio Martínez Adame, Víctor L. Urquidi colaboró en los trabajos editoriales de la primera revista mexicana especializada en economía, política económica y desarrollo, El Trimestre Económico, la cual, había sido fundada en 1934 por los primeros dos personajes señalados líneas arriba. De 1949 a 1957, Urquidi dirigió la revista.

En reconocimiento a casi dos muy fructíferas décadas de trabajo profesional, académico y editorial, Víctor L. Urquidi ingresó a El Colegio Nacional en octubre de

2 Víctor L. Urquidi (1996), "Reconstruction vs. Development: the IMF and the World Bank", capítulo 3 del libro editado por Kirschner, Orin (1996), The Bretton Woods-GATT System: Retrospect and Prospect After Fifty Years, Routledge, London \& New York.

3 Joseph Hodara (2008), "Prebisch y Urquidi: vidas paralelas", Araucaria. Revista Iberoamericana de Filosofía, Política y Humanidades, No 19. Primer semestre de 2008, pp. 46-63. También disponible al teclear en cualquier buscador de internet la siguiente dirección: http://institucional. us.es/revistas/Araucaria/Año\%2010\%20\%20N\%2019\%20\%202008/Joseph\%20Hodara.pdf 
1960. En su discurso de ingreso, publicado poco después por El Trimestre Económico, entre otras importantes ideas, expresó las siguientes: ${ }^{4}$

Se ha escrito y hablado mucho sobre si la Economía es la ciencia de la riqueza y el bienestar, o si constituye más bien la ciencia de la pobreza y el pesimismo. Según muchos, no llega a ciencia siquiera, sino que es un simple método o arte. En realidad, es ambas cosas: ciencia y arte. Es ciencia en tanto constituye una disciplina que pretende explicar de manera sistemática las causas de ciertos fenómenos y sus consecuencias, con apoyo en observaciones objetivas. Es arte en tanto descansa, especialmente por lo que respecta a su fase normativa, y también a la inductiva, en el falible juicio subjetivo del hombre y en su habilidad particular...

Salvo en la abstracción científica, la Economía es en realidad Economía Política, o sea economía normativa, uno de los aspectos de la organización de la sociedad. En este sentido, la Economía está al servicio de la Política y todo economista es poseedor consciente o inconsciente de un prejuicio. La Ciencia Económica podrá ser neutral en cuanto constituya una serie de principios abstractos de alcance limitado; mas en manos del economista no puede ser sino un antecedente de una política económica que persiga un fin social determinado...

Podría entonces la Ciencia Económica convertirse en un instrumento más eficaz de la planificación del porvenir de la humanidad. De otra manera, la Economía estará condenada a volverse el arte de la explicación a posteriori, con frecuencia de carácter apologético, en lugar de ser una de las guías del buen gobierno.

La heterodoxia de Urquidi estuvo erigida en el conocimiento profundo de la ortodoxia económica, en la indiscutible influencia que en él también tuvo la Teoría General de la Ocupación, el Interés y el Dinero de Keynes, en su crítica lectura de Marx, y en el ascenso y la promoción propia de las ideas latinoamericanas sobre el desarrollo y el subdesarrollo económicos, ingredientes imprescindibles de aquella emergente aventura llamada Cepal que, con buen tino y cierta saudade, Celso Furtado llamara la fantasía organizada. 5 Puede afirmarse, mediando las actualizaciones y ajustes educados que se juzguen pertinentes, que la heterodoxia de Urquidi es la heterodoxia que hoy necesitamos, basada no en un eclecticismo superficial ni forzado, menos aún en la descalificación huera de cualquier perspectiva disciplinaria que confronte las ideas, convicciones y argumentos propios, sino en el conocimiento riguroso tanto de las diversas corrientes de la disciplina económica, de la historia económica, de los contextos institucionales específicos, como de sus respectivas capacidades analíticas y normativas ante los acontecimientos económicos de la realidad contemporánea, nacional e internacional, y los inciertos o probables escenarios de desenlace futuro. En

4 Víctor L. Urquidi (1961), "La responsabilidad de la Economía y del economista", discurso de ingreso a El Colegio Nacional, El Trimestre Económico, número 109. También disponible al teclear en cualquier buscador de internet la siguiente dirección: http://aleph.academica.mx/jspui/ bitstream/56789/7414/1/DOCT2064807_ARTICULO_1.PDF

5

Celso Furtado (1985), La fantasía organizada, Buenos Aires, Editorial Eudeba, 1988. 
suma, se trata de una suerte de insatisfacción metodológica que genere polémicas provechosas, trascendentes, útiles para el avance disciplinario y el mejoramiento de las políticas y el quehacer público de los economistas.

Otra contribución más en la caleidoscópica obra de Urquidi, se encuentra también en ese mismo crítico y muy inspirador discurso de ingreso a El Colegio Nacional, en 1960. Me disculpo por volver a citarlo in extenso, 6 pero lo considero imprescindible en mi argumentación acerca de la hondura y la vigencia de su legado:

La situación en que se encuentra la Economía en su aspecto teórico merece una crítica más insistente, de la que no puede librarse ninguna escuela, ni aun la marxista. Gran parte de lo que se expone y enseña en cuanto economía teórica, en cualquier parte del mundo, carece casi de todo sentido, cuando no es lucubración inútil por su falta de relación con la realidad. Se elaboran, sostienen y repiten teorías y teoremas que en su origen no fueron sino justificaciones de una política económica determinada o de un ideal jamás cumplido. Sin embargo, esas teorías se siguen proclamando como si fueran verdades científicas. Los libros de texto, los tratados sobre Economía y los artículos doctos han sido emponzoñados por una seudo-ciencia económica que el más débil sentido de responsabilidad hacia la sociedad actual obligaría a descartar por superflua.

... Son ideas, conceptos, lo que más necesita la Economía de hoy. Al pensamiento fundamental debe acoplarse la teoría, con la ayuda de las disciplinas matemáticas y otras afines, en lugar de que ocurra al revés: que de las formulaciones matemáticas se deduzcan conclusiones de política e ideología económicas. La Economía siempre ha sido, y deberá seguir siendo, una ciencia al servicio de las ideas...

... En Economía Política los acontecimientos no son inevitables, ni los benéficos ni los perjudiciales. Pero hay que poder medir las consecuencias de distintas alternativas... los conceptos pertinentes a la Economía Política descansan en premisas institucionales y no tienen validez sino en conjunción con las premisas de que se trate. La mayor parte de ellas son políticas. Mas las hay también de otro orden, surgidas del desarrollo histórico de la economía mundial y de las sociedades humanas...

... La enseñanza de la Economía no ha alcanzado a ser suficientemente sólida. Una hojeada a los planes de estudio de nuestros centros universitarios revela que están anquilosados, y eso sin entrar a examinar el contenido de cada asignatura, que rara vez se ha renovado. El pensamiento teórico, aunque en ocasiones haya reproducido las enseñanzas venidas de otras partes, ha sido en gran medida estático, o sólo ha manifestado ciertos destellos.

A más de 55 años de haberse escrito y publicado el multicitado discurso, los argumentos de Urquidi tienen pertinencia hoy y razones de sobra. En la actualidad, la inconformidad acerca de las capacidades heurística, exegética y normativa de las versiones predominantes de la denominada doctrina económica ortodoxa, se ha difundido. Aún y cuando en su lugar no hayan prosperado explicaciones ni propuestas profusamente influyentes o redondamente acabadas, pero sí potencialmente más

6 Víctor L. Urquidi (1961), "La responsabilidad de la Economía y del economista”, op. cit. 
eficaces en términos analíticos y normativos, Urquidi mantiene abierta la puerta a las diversas expresiones de la heterodoxia económica, de importantes hibridaciones conceptuales, así como a la propagación, esperanzadamente no dogmática, de formulaciones inspiradas en diversas corrientes contradictorias, incompletas, en permanente disputa, del pensamiento económico contemporáneo, incluidas por supuesto, las autocríticas y los desarrollos teóricos al interior de la propia matriz de la economía convencional. Quien enfrente preocupaciones disciplinarias, profesionales o políticas similares a las aquí aludidas, Urquidi también es una inspiradora influencia para pensar con libertad y discernir con responsabilidad, entre las mejores opciones académicas y prácticas, relacionadas con la nada fácil pero apasionante tarea de la formación de economistas para el siglo XXI mexicano.

Como miembro del Club de Roma, Urquidi prologó la versión en español del famoso libro Los límites del crecimiento. ${ }^{7}$ Allí expresa que el problema central de ese momento y, a partir de entonces, también del largo y muy largo plazos, el problema epocal, dirían algunos, es:

... el de la capacidad del planeta en que convivimos para hacer frente, más allá del año 2000 y bien entrado el siglo XXI, a las necesidades y modos de vida de una población mundial siempre creciente, que utiliza a tasa acelerada los recursos naturales disponibles, causa daños con frecuencia irreparables al medio ambiente y pone en peligro el equilibrio ecológico global, todo ello en aras de la meta del crecimiento económico, que suele identificarse con bienestar.

Su metódico y sistemático estudio de las interrelaciones del análisis económico con el análisis institucional, demográfico y ambiental, entre otras consecuencias, influyeron en la conformación del Consejo Nacional de Población (Conapo) en 1974, así como en la formulación y puesta en vigor de una entonces explícita y progresista política demográfica de planificación familiar en el país. Participó en los trabajos que condujeron a la promulgación de la Ley General del Equilibrio Ecológico y la Protección al Ambiente (LGEEPA) en enero de 1988, así como en su reforma, realizada en 1996, donde asesoró a los economistas que, desde el Instituto Nacional de Ecología (INE) y la entonces Secretaría de Medio Ambiente, Recursos Naturales y Pesca (Semarnap), redactaron el capítulo IV, sección III "Instrumentos Económicos", de la propia LGEEPA, específicamente sus artículos 21, 22 y 22bis, que apoyan la mejor y más eficiente implementación del ordenamiento jurídico-ambiental referido. Allí, 8 a la letra se dice:

Se consideran instrumentos económicos los mecanismos normativos y administrativos de carácter fiscal, financiero o de mercado, mediante los cuales las personas asumen los beneficios y costos ambientales que generen sus actividades económicas, incentivándolas a realizar acciones que favorezcan el ambiente.

7 Víctor L. Urquidi (1972), “Allende el año 2000”, Prólogo a la edición en español del libro: Meadows, Dennis L. et al. (1972), Los límites del crecimiento, México, Fondo de Cultura Económica.

8 Ley General del Equilibrio Ecológico y la Protección al Ambiente (LGEEPA, 1988, 1996, 2012), artículo 22, sección III, capítulo IV. 
Fue creador del Centro de Estudios Económicos y Demográficos de El Colegio de México y participante clave en la puesta en marcha del programa académico internacional, con énfasis profesionalizante, Leadership for Environment and Development (LEAD), alojado también en El Colegio de México. Sin duda alguna, Urquidi fue el precursor de la economía ambiental en México y formador o referencia obligada de los economistas ambientales del país. Su comprehensiva mirada hacia la importancia y la responsabilidad de su profesión y de sus colegas economistas no debe olvidarse:

Es a todas luces conveniente que el economista mexicano actúe en la vida pública y en la actividad privada aportando sus conocimientos especializados y ejerciendo influencia... pero igualmente importante es que el economista tenga oportunidad, y muestre deseos, de desenvolverse por los senderos científicos de su carrera. ${ }^{9}$

Por todo lo anteriormente expresado, Víctor L. Urquidi debiera ser ejemplo tanto para quienes toman decisiones económicas y de política económica hoy día, así como para quienes pertenecen a las actuales generaciones de formadores de economistas y de economistas en formación.

\section{Conclusión}

El estudio de los procesos de contaminación ambiental, degradación ecológica y de pérdida neta de recursos naturales correlacionados con el crecimiento económico y la política económica y demográfica, fueron las preocupaciones centrales de Víctor L. Urquidi durante poco más de tres décadas, desde finales de los años sesenta. Con estas preocupaciones en mente, fue que, reconociendo las importantes contribuciones de las teorías del desarrollo de Gunnar Myrdal y Celso Furtado, echaba de menos en ellas, consideraciones explícitas sobre los crecientes y acumulativos costos ambientales del desarrollo económico. Para Urquidi, la teoría y la práctica del desarrollo se había concentrado en un importante proceso de inversión física para incrementar la capacidad productiva en diferentes actividades económicas de México y de otras economías asiáticas, europeas y latinoamericanas, señaladamente mediante la industrialización, la modernización económica general y activas políticas que mejoraron los niveles de educación y de cobertura en servicios de salud, hasta la crisis financiera internacional de los años ochenta del siglo pasado. Criticó que, paralelamente, nunca haya sido una preocupación genuina, de las propias políticas de desarrollo, el conjunto de impactos ambientales adversos, consustanciales al mismo. De allí la importancia que le otorgó a los estudios, las discusiones y a la construcción de instituciones relacionadas con el desarrollo sustentable desde 1987. Nuevo discurso que, de manera explícita, pretendía expresar, con evidencias duras, los frutos del desarrollo en términos de bienestar social neto inter-temporal, al poner el acento en los asuntos ambientales públicos y privados-, nacionales e internacionales. Al respecto, Urquidi señaló que de no trascenderse el acotado margen de acción y la limitada influencia de las "políticas

9 Víctor L. Urquidi (1961), “La responsabilidad de la Economía y del economista”, op. cit. 
ambientales específicas", con mucha dificultad podría ingresarse a un planteamiento más sistémico y desafiante de política para el desarrollo sustentable. 10

En el mismo artículo apenas referido, Urquidi señala que debe hacerse una medición del crecimiento económico distinta, ajustarlo ambientalmente y auto-exigirse la medición del desarrollo sustentable. Al respecto, el Instituto Nacional de Estadística y Geografía (INEGI) publica, desde 1996, el producto interno neto ecológico (PINE), al deducir del producto interno bruto (PIB), la depreciación del capital fijo, así como los costos por agotamiento de recursos naturales y por la contaminación ambiental, en el marco del Sistema de Cuentas Económicas y Ecológicas de México (SCEEM). Los actuales economistas ambientales, debemos analizar, discutir, usar, y volver a discutir las cifras publicadas por INEGI al respecto. También debemos estudiar, discutir, usar y criticar los fundamentos y desarrollos teóricos de la economía ambiental, la economía ecológica y la economía de los recursos naturales. En suma, debemos tener un talante analítico y normativo de inconformidad, lo más ilustradamente posible, para inducir y participar en polémicas provechosas que, tenue pero crecientemente, vayan influyendo en la vida pública nacional. Esta sería la mejor manera de mantener vivo durante mucho tiempo más, el espíritu riguroso y la conducta responsable de Víctor L. Urquidi.

\section{Anexo}

\section{Enmienda redactada y leída por Víctor L. Urquidi}

Proceedings and Documents of United Nations Monetary and Financial Conference, Bretton Woods, New Hampshire, july 1-22, 1944, vol. II.

\section{Document 306}

UNITED NATIONS MONETARY AND FINANCIAL CONFERENCE

Statement by Delegation of Mexico at Meeting of Commission II, July 11, 1944

Mr. Chairman, Fellow Delegates:

On behalf of the Mexican Delegation, may I be allowed to make a brief explanatory statement on the alternative provision submitted by us which is now before you.

It may appear to some of you that our proposal would rather hamper the Bank's reconstruction operations during the first few years. But I wish to assure you, gentlemen, that it is very far from our purpose to place obstacles in the way of reconstruction. We are fully aware of the damage that the war has done to the productive capacity of our Allies in Europe and in Asia, and we realize also that, once liberated, the territories now occupied by our enemies will require a great deal of capital in order to be set afoot again. We are no less aware of the direct sacrifices under gone by all those nations. Therefore, it is not with a spirit of denying them a substantial measure, of the Bank's resources that we have introduced this - to our mind-important amendment.

10 Víctor L. Urquidi (1999), “Dimensiones del desarrollo sustentable y el caso de México”, en Alejandro Nadal (2007), editor, Obras Escogidas de Víctor L. Urquidi, primer volumen, El Colegio de México. 
Our reasons for asking you to provide that "reconstruction" and "development" be put on the same footing are threefold:

First, we believe that the agreement we are to reach here is to be embodied in a permanent, and not in a provisional, inter national instrument. Therefore, it seems to us inappropriate that the document should not contain an equal emphasis on the two great purposes of the Bank, namely, to facilitate reconstruction and development. In the very short run, perhaps reconstruction will be more urgent for the world as a whole, but in the long run, Mr. Chairman-before we are all too dead, if I may say so- development must prevail if we are to sustain and increase real income everywhere. Without denying the initial importance of reconstruction, we ask you not to relegate or postpone development.

Secondly, we believe that we and other nations not actually in need of funds for reconstruction, can greatly assist in the reconstruction of those who do necessitate it, provided our economies be developed more fully at the same time as the rehabilitation of the war torn nations takes place. We have resources which are still untapped. A large part of our population has not yet attained an adequate standard of living. And yet we have not hesitated to throw in our lot with our Allies, disregarding temporarily our own wide domestic problems. If we tackle these-and for that we require sums of capital we do not dispose of at home-we will undoubtedly benefit not only ourselves but the world as a whole, and particularly the industrial nations, in that we shall provide better markets for them and better customers. We submit, there fore, that capital for development purposes in our countries is as important for the world as is capital for reconstruction purposes.

Third and last-and we again wish to emphasize that it is with no unfriendly spirit that we make this reference-we should like to call your attention to an important provision of the draft (Article II, Section 5-A), which states that payments in gold shall be graduated according to a schedule that shall take into account the adequacy of the gold and free foreign exchange holdings of each member country. We believe that, having in mind the position in which the war devastated countries are, this is only fair; and we have no intention whatever of grudging one ounce of our contribution in gold. But since we happen to have unprecedented holdings of gold and foreign exchange-we speak for the great majority of Latin American nations - and since we feel that we have before us an opportunity of devoting part of our holdings to the import of capital goods for our development, it is our considered opinion that in contributing part of them, ungrudgingly, to the Bank, for the benefit of all the nations constituting it, we should desire at least the assurance that our requests for capital for development purposes shall, in the words of our amendment, be given equal consideration as is given to reconstruction projects, and, further, the assurance that the resources and facilities of the Bank shall always be made available to the same extent for either kind of project.

We do wish to make it perfectly clear, however, Mr. Chairman, that we do not desire to impose on the Bank a rigid fifty-fifty rule. We believe some discretion on the Bank's part should be provided for. Furthermore, what we ask is only that the Bank's resources and facilities be made available. Thus, in the event that countries requesting loans for development purposes do not use up the resources and facilities made available to them, countries requiring loans for reconstruction projects could have a claim on the unused funds.

In conclusion, may we emphasize that we do not contemplate a rigid interpretation of the phrase "to the same extent", but that we do think it is a principle which should be embodied in the instrument we are endeavoring to draw up. We are perfectly willing to accept a better wording of our proposed amendment, so long as the same principle is preserved in it. 


\section{Bibliografía}

FuRTADO, Celso (1985), La fantasía organizada, Buenos Aires, Editorial Eudeba, 1988.

HODARA, Joseph (2008), "Prebisch y Urquidi: vidas paralelas", Araucaria. Revista Iberoamericana de Filosofía, Política y Humanidades, No 19. Primer semestre de 2008, pp. 46-63. También disponible al teclear en cualquier buscador de internet la siguiente dirección: http://institucional.us.es/revistas/ Araucaria/Año\%2010\%20\%20N%2019\%20\%202008/Joseph\%20Hodara.pdf

LGEEPA $(1988,1996,2012)$, Ley General del Equilibrio Ecológico y la Protección al Ambiente...

United Nations, Proceedings and Documents of United Nations Monetary and Financial Conference,

Bretton Woods, New Hampshire, july 1-22, 1944. También disponible al teclear en cualquier buscador de internet la siguiente dirección: https://fraser.stlouisfed.org/docs/publications/books/1948_state_ bwood_v2.pdf

URQUIDI, Víctor L. (1961), "La responsabilidad de la Economía y del economista”, discurso de ingreso a EI Colegio Nacional, El Trimestre Económico, número 109. También disponible al teclear en cualquier buscador de internet la siguiente dirección: http://aleph.academica.mx/jspui/bitstream/56789/7414/1/ DOCT2064807_ARTICULO_1.PDF

URQUIDI, Víctor L. (1972), “Allende el año 2000”, Prólogo a la edición en español del libro: Meadows, Dennis L. et al. (1972), Los límites del crecimiento, México, Fondo de Cultura Económica.

URQUIDI, Víctor L. (1996), "Reconstruction vs. Development: the IMF and the World Bank", capítulo 3 del libro editado por Kirschner, Orin (1996), The Bretton Woods-GATT System: Retrospect and Prospect After Fifty Years, Routledge, London \& New York.

URQUIDI, Víctor L. (1999), “Dimensiones del desarrollo sustentable y el caso de México”, en Alejandro Nadal (2007), editor, Obras Escogidas de Víctor L. Urquidi, primer volumen, El Colegio de México. 